\title{
Chemical characterization of basil (Ocimum spp.) based on volatile oils
}

\author{
Roberto F. Vieira ${ }^{1}$ and James E. Simon ${ }^{2, *}$ \\ 1 Embrapa, Cenargen, Caixa Postal 02372, Brasilia, DF, 70770-990, Brazil \\ 2 New Use Agriculture and Natural Plant Products Program, Cook College and the New Jersey Agriculture Experiment Station, \\ Rutgers University, 59 Dudley Road, New Brunswick, NJ 08901-8520, USA
}

Received 10 June 2003; Revised 7 January 2004; Accepted 29 June 2004

\begin{abstract}
Essential oils extracted from basil (Ocimum spp.) by steam distillation are used to flavor foods, oral products, in fragrances and in traditional medicines. The genus Ocimum contains around 30 species native to the tropics and subtropics, with some species naturalized and/or cultivated in temperate areas. Inter- and intraspecific hybridization have created significant confusion in the botanical systematics of the Ocimum genus. The taxonomy of $O$. basilicum is further complicated by the existence of numerous varieties, cultivars and chemotypes within the species that do not differ significantly in morphology. The composition of volatile oil constituents was used to characterize the diversity among the most economically important Ocimum species. Using principal component analysis on the aromatic volatile oils, the Ocimum accessions could be separated into five groups, which do not correspond to the different species: (1) citral-spathulenol accessions; (2) linalool-rich accessions; (3) methylchavicol-rich accessions; (4) linalool-methylchavicol accessions; and (5) methyl $(E)$-cinnamate-rich accessions. The fact that the groups of Ocimum species are based on morphological characteristics does not correspond to the groups based on volatile oil constituents. Copyright @ 2006 John Wiley \& Sons, Ltd.
\end{abstract}

KEY WORDS: Ocimum; O. basilicum; O. $\times$ citriodorum; O. americanum var. americanum; O. americanum var. pilosum; O. minimum; O. kilimandscharicum; essential oils; infraspecific variability, chemotaxonomy

\section{Introduction}

Basil (Ocimum basilicum L.) leaves are used fresh and dried as flavorings or spices in sauces, salad dressings, vegetables, vinegar and confectionery products. ${ }^{1}$ Basil is also an important ornamental plant, with a variety of leaf and inflorescence shapes, sizes and colors. Besides the commercial basil, many other species of Ocimum are used in traditional ceremonies and medicines, mainly in their country of origin. ${ }^{2-4}$ Extracted essential oils from basil are used to flavor foods and dental and oral products. ${ }^{1}$ Basil volatile oils have also been show to contain biologically active constituents that are insecticidal, nematicidal, fungistatic and have antimicrobial properties. $^{5}$

More than 40 types of basil available in North America $^{6}$ have been described. Most varieties available and used belong to $O$. basilicum, the most cultivated species. Darrah ${ }^{7}$ classified $O$. basilicum varieties into four groups: (1) tall slender types, which included the variety 'Sweet Basil'; (2) large-leaved, robust types, such as the

\footnotetext{
* Correspondence to: J. E. Simon, New Use Agriculture and Natural Plant Products Program, Cook College and the New Jersey Agriculture Experiment Station, Rutgers University, 59 Dudley Road, New Brunswick, NJ 08901-8520, USA

E-mail: jesimon@aesop.rutgers.edu

Contract/grant sponsor: Brazilian Agricultural Research Corporation.

Contract/grant sponsor: Purdue University.

Contract/grant sponsor: New Use Agriculture and Natural Plant Products

Program, Rutgers University.
}

variety 'Lettuce Leaf'; (3) dwarf types, small-leaved, including the variety 'Bush' basil; and (4) compact types, described as a botanical variety, $O$. basilicum var. thyrsiflora, commonly called Thai basil. Later, Darrah ${ }^{8}$ included three additional groups: (5) O. basilicum var. purpurascens, the purple basil types with a sweet flavor; (6) dark opal type, a possible hybrid between $O$. basilicum and $O$. forskolei, which has lobed purple leaves with sweet basil plus clove flavor; and (7) citriodorumtype, including lemon flavor basil.

Basil (O. basilicum) varieties have been selected and developed over many years for a variety of purposes. The culinary basils were selected for their leaf shape, size, aroma and flavor. In the sweet basil group are many green basil varieties, rich in a combination of linalool, methylchavicol and 1,8-cineole. The most important ones used in fresh culinary herbs include 'Italian Large Leaf', 'Genovese', and 'Sweet basil'. Basils were also selected and modified to enhance their ornamental qualities, focusing on such traits as plant height, leaf color and shape, inflorescence length, color and compactness. Examples include the varieties 'Bush', selected for its dwarf form, 'Purple Ruffles', selected for its leaf color, 'Siam Queen' for its beauty and 'Sweet Dani Lemon Basil' for its citral aroma and large leaf. Centuries of selection of basil for distinct aromas also led to the establishment of many chemotypes rich in a variety of volatile oil compounds, including citral, linalool, methylchavicol, eugenol, geraniol and methylcinnamate. 
Several types of basils are also produced for their extractable essential oils, which are traded in the international essential oil market, each derived from different cultivar or chemotype of $O$. basilicum, and classified according to their geographic origin. ${ }^{1,9}$ The European type (Italy, French or sweet basil), considered to be the highest quality and producing the finest odor, contains linalool and methylchavicol as the major constituents. ${ }^{1}$ Egyptian basil is very similar to the European, but with a higher relative percentage of methylchavicol. The Reunion type, from the Reunion and Comoro Islands, Thailand, Madagascar and Vietnam, is characterized by its high concentrations of methylchavicol. ${ }^{9}$ Methylcinnamate-rich basil has been commercially produced in Eastern Europe, ${ }^{1}$ India, Guatemala and Pakistan. ${ }^{9}$ A basil from Java, ${ }^{1}$ and Russia and North Africa $^{9}$ has been reported to be a eugenol-rich basil.

The occurrence of interspecific hybridization and polyploidy within this genus ${ }^{10}$ of about 30 species $^{11}$ has created taxonomic confusion and challenges to understanding the genetic relationship between the multitude of basils. ${ }^{12}$ Taxonomy of basil ( $O$. basilicum) is also complicated by the existence of numerous varieties, cultivars and chemotypes within the species that do not differ significantly in morphology. ${ }^{1}$ Hybridization of many different species and varieties, and the widespread use of commercial variety names, have obscured important information such as variation in chemistry and chromosome number. Paton and Putievsky ${ }^{13}$ reported that there is no clear morphological discontinuity between $O$. basilicum varieties.

A system of standardized descriptors, including volatile oil, was proposed by Paton and Putievsky ${ }^{13}$ to allow easy communication and correct identification of the different forms of $O$. basilicum. Molecular markers using RAPD have been used successful in assessing genetic diversity and distinguishing genetic relatedness among a wide number of Ocimum spp. ${ }^{14,15}$

Principal component analysis (PCA) has been successfully used in many aromatic and polymorphic species, such as Mentha and Thymus, ${ }^{16-18}$ and has been associated with RAPD markers. ${ }^{14,15,19,20}$ Our results with O. gratissimum ${ }^{14}$ showed that the use of principal component analysis can be an important tool for working on multivariate data.

The objective of this research was to chemically characterize the volatile oil constituents in an Ocimum germplasm collection and to access the relative use of this parameter as a taxonomical descriptor.

\section{Material and Methods}

\section{Plant Material}

Twenty-six accessions (Table 1) of Ocimum basilicum
(15), O. americanum var. americanum (2), $O$. americanum var. pilosum (1), O. minimum (3), O. $\times$ citriodorum (4), and one hybrid between $O$. basilicum and $O$. kilimandscharicum [obtained from Richters Seed Co., Goodwood, Ontario, Canada; Companion Plants, Athens, OH, USA; Purdue University, West Lafayette, IN, USA; Kew Gardens, London, UK; US Department of Agriculture (USDA), Plant Introduction Station, Ames, IA, USA; Brazilian Agricultural Research Corporation (Embrapa), Brasília, Brazil; and Nichols Garden, Albany, OR, USA] were grown at Purdue University Research Station, West Lafayette (IN, USA) in a randomized complete-block experimental design, with four plants in each of the three replications. The above-ground biomass of each individual plant from each block was harvested at full bloom, bulked, placed in a paper bag and dried in a forced-air drier at $38^{\circ} \mathrm{C}$ for 15 days for oil analysis. Voucher specimens of each accession included in our experiments were collected, dried and stored at the Ralph Kriebel herbarium (PUL), Purdue University. The taxonomic identification was conducted in collaboration with Dr A. Paton from the Royal Botanical Garden, Kew, London, Dr A. Tucker from Delaware State University and Dr U. Albuquerque from Universidade Federal de Pernambuco, Brazil. Vouchers specimens were sent to Kew Gardens (K), Delaware State University (DOV) and Cenargen, Embrapa (CEN) herbaria for permanent collection.

\section{Volatile Oil Analysis}

The volatile oil was extracted from dried samples in a 21 flask with distilled water $(1: 15 \mathrm{w} / \mathrm{v})$ using a Clevenger apparatus, as previously described. ${ }^{21}$ Volatile oil samples were analyzed in a Varian 3700 gas chromatograph equipped with a flame ionization detector (FID) and an electronic 4270 integrator. A fused silica capillary column $(30 \mathrm{~m} \times 0.25 \mathrm{~mm}$ i.d.) with an RSL-200 (Altech, $5 \%$ phenyl, $95 \%$ methylpolysiloxane) bonded phase was used. Helium was used as a carrier gas and the oven temperature was held isothermal at $80^{\circ} \mathrm{C}$ for $2 \mathrm{~min}$ and then programmed to increase at $3{ }^{\circ} \mathrm{C} / \mathrm{min}$ to $160{ }^{\circ} \mathrm{C}$. This gave a complete elution of all peaks (using a sensitivity of $10^{-10}$, attenuation 16). The injector and detector temperatures were 180 and $300{ }^{\circ} \mathrm{C}$, respectively. Standards samples of oil constituents ( $p$-cymene, 1,8-cineole, terpinen-4-ol, methylchavicol, geraniol, thymol, eugenol, methyleugenol and $\beta$-caryophyllene) were run at the same conditions. Identification of essential oil constituents was confirmed by GC/MS using a Finningan 4000; the carrier gas was helium with a column pressure of $10.5 \mathrm{psi}$ and split vent of $40 \mathrm{ml} / \mathrm{min}$; the oven program was $80^{\circ} \mathrm{C}$ for $2 \mathrm{~min}$ and then increasing at $3{ }^{\circ} \mathrm{C} / \mathrm{min}$ to $160^{\circ} \mathrm{C}$; injection temperature was $225^{\circ} \mathrm{C}$. The MS conditions include ionization voltage, $70 \mathrm{eV}$; emission 
current, $40 \mathrm{~mA}$; scan rate, $1 \mathrm{scan} / \mathrm{s}$; mass range, 40-500 $\mathrm{Da}$; ion source temperature, $160{ }^{\circ} \mathrm{C} .{ }^{21}$

\section{Data Analysis}

Statistical analysis of volatile oil constituents was performed using SAS software (SAS Institute Inc., Cary, NC, USA) procedures. Principal component analysis (PCA) was performed to display the relationship among accessions in terms of their position relative to three coordinate axes. All PCA was carried out using SAS (SAS Institute Inc., Cary, NC, USA) software procedures, and drawn using Sigma Plot (Jandel Corporation, San Rafael, CA, USA).

\section{Results and Discussion}

Twenty-seven volatile oil constituents (Tables 1-5) were identified across the 26 Ocimum accessions (Table 1).
The essential oil content varied from $0.27 \%$ in $O$. americanum var. pilosum (ot21) to 2.47 and $2.8 \%$ in $O$. americanum var. americanum (ot5) and 'African blue' (ot50), respectively. The shortest plants were from $O$. minimum (ot34, ot35 and ot60), each reaching heights of only $28.3,24.8$ and $30.8 \mathrm{~cm}$, respectively. In contrast, plants of $O$. basilicum reached heights of from $33.8 \mathrm{~cm}$ in 'Green Ruffles' (ot41) to $72.3 \mathrm{~cm}$ in 'Sweet Dani Lemon Basil’ (ot70).

Essential oil content in $O . \times$ citriodorum ranged (as a percentage dry weight) from 0.36 (ot58) to $0.39 \%$ (ot53) to 1.64 and $1.12 \%$, for 'Lemon Mrs. Burns' (ot61) and 'New Guinea' (ot62), respectively (Table 1). The ratios of total volatile oil in 'Sweet Dani' (ot70) and 'Lemon Mrs. Burns' (ot61), both considered O. basilicum with strong lemon ancestry basil types, ${ }^{11}$ were consistent with the literature.

'Sweet Dani Lemon Basil' (ot70) contained the highest citral concentration $(33.6 \%)$; however, the relative amount was much lower than the $68 \%$ citral reported by Morales and Simon. ${ }^{22}$ Grayer et al. $^{23}$ reported a citral

Table 1. List of Ocimum accessions, origin, growth and essential oil accumulation, grown at Purdue University (West Lafayette, IN, USA)

\begin{tabular}{|c|c|c|c|c|c|}
\hline $\begin{array}{l}\text { Species } \\
\text { accessions }\end{array}$ & Origin & $\begin{array}{l}\text { Height } \\
(\mathrm{cm})\end{array}$ & $\begin{array}{l}\text { Total fresh } \\
\text { weight }(\mathrm{g})\end{array}$ & $\begin{array}{c}\text { Total dry } \\
\text { weight (g) }\end{array}$ & $\begin{array}{c}\text { Essential oil } \\
\text { (\% dry weight) }\end{array}$ \\
\hline \multicolumn{6}{|c|}{ O. americanum var. americanum } \\
\hline ot $5^{\mathrm{a}}$ & Kew90157, Kew Garden & $45.3 \pm 1.7^{\mathrm{b}}$ & $502.3 \pm 117$ & $112.2 \pm 25$ & $2.47 \pm 0.2$ \\
\hline ot32 & Brasilia, Brazil & $34.3 \pm 2.4$ & $266.3 \pm 17$ & $69.8 \pm 4$ & $1.68 \pm 0.4$ \\
\hline \multicolumn{6}{|c|}{ O. americanum var. pilosum } \\
\hline ot21 & PI414204, USDA & $46.3 \pm 3.8$ & $494.5 \pm 70$ & $112.4 \pm 16$ & $0.27 \pm 0.1$ \\
\hline \multicolumn{6}{|l|}{ O. basilicum } \\
\hline ot2 & Kew7711, Kew Garden & $48.5 \pm 1.3$ & $301 \pm 116$ & $79 \pm 28$ & $0.87 \pm 0.2$ \\
\hline ot38 & Dark Opal, Richters Co. & $44.8 \pm 3.6$ & $391.3 \pm 77$ & $59.7 \pm 12$ & $1.1 \pm 0.2$ \\
\hline ot 40 & Purple Ruffles, Richters Co. & $34.8 \pm 2.6$ & $218.8 \pm 50$ & $30.1 \pm 7$ & $0.58 \pm 0.4$ \\
\hline ot41 & Green Ruffles, Richters Co. & $33.8 \pm 5.3$ & $490.8 \pm 194$ & $68.6 \pm 25$ & $0.83 \pm 0.2$ \\
\hline ot43 & Mammoth, Richters Co. & $42 \pm 9.1$ & $672.3 \pm 345$ & $106.8 \pm 56$ & $0.75 \pm 0.2$ \\
\hline ot 44 & Sweet, Richters Co. & $49 \pm 5.6$ & $544.5 \pm 255$ & $94.6 \pm 45$ & $1.12 \pm 0.2$ \\
\hline ot 45 & Sweet Fine, Richters Co. & $45.5 \pm 0.7$ & $403.5 \pm 103$ & $71 \pm 13$ & $0.77 \pm 0.3$ \\
\hline ot 49 & Cinnamon, Richters Co. & $46.5 \pm 5.2$ & $554.3 \pm 163$ & $97.2 \pm 65$ & $1.54 \pm 0.3$ \\
\hline ot56 & Thai, Richters Co. & $38 \pm 3.3$ & $538.3 \pm 164$ & $96.5 \pm 24$ & $0.71 \pm 0.1$ \\
\hline ot57 & Thai Siam Queen, Richters Co. & $38.3 \pm 5.6$ & $544.3 \pm 58$ & $93.6 \pm 3$ & $0.70 \pm 0.1$ \\
\hline ot61 & Lemon Mrs Burns, Companion & $59 \pm 5.4$ & $754.3 \pm 272$ & $150.1 \pm 59$ & $1.64 \pm 0.2$ \\
\hline ot69 & Comoro, Purdue & $61 \pm 1.4$ & $697.9 \pm 205$ & $168.3 \pm 35.6$ & $1.04 \pm 0.3$ \\
\hline ot70 & Sweet Dani Lemon Basil, Purdue & $72.3 \pm 4.0$ & $761.5 \pm 132$ & $176 \pm 38.4$ & $0.54 \pm 0.1$ \\
\hline ot71 & $\operatorname{Methyl}(E)$-cinnamate basil, Purdue & $38.9 \pm 3$ & $462.8 \pm 122$ & $102.9 \pm 25.3$ & $1.37 \pm 0.3$ \\
\hline ot72 & Linalool basil, Purdue & $58.5 \pm 3.3$ & $638.2 \pm 236$ & $137.4 \pm 46.2$ & $1.13 \pm 0.3$ \\
\hline \multicolumn{6}{|c|}{ O. basilicum cv. Dark Opal $\times O$. kilimandscharicum } \\
\hline ot50 & African Blue, Richters Co. & $56.3 \pm 1.7$ & $636.3 \pm 185$ & $141.6 \pm 38.2$ & $2.8 \pm 0.1$ \\
\hline \multicolumn{6}{|c|}{ O. $\times$ citriodorum } \\
\hline ot53 & Lemon, Richters Co. & $46 \pm 2.2$ & $354.5 \pm 109$ & $93.2 \pm 29.3$ & $0.39 \pm 0.2$ \\
\hline ot58 & Lemon, Companion & $48.8 \pm 3.2$ & $499.2 \pm 117$ & $109.1 \pm 18.4$ & $0.36 \pm 0.1$ \\
\hline ot62 & New Guinea, Companion & $32 \pm 2.5$ & $303 \pm 43$ & $64.2 \pm 12.2$ & $1.12 \pm 0.4$ \\
\hline ot66 & Lemon, Nichols Garden Nursery & $45.5 \pm 4.0$ & $406.8 \pm 143$ & $99.9 \pm 35.9$ & $0.38 \pm 0.1$ \\
\hline \multicolumn{6}{|l|}{ O. minimum } \\
\hline ot34 & Bush, Richters Co. & $28.3 \pm 2.6$ & $471 \pm 103$ & $82 \pm 11.4$ & $1.06 \pm 0.2$ \\
\hline ot35 & Spicy globe, Richters Co. & $24.8 \pm 5.0$ & $433 \pm 167$ & $64.1 \pm 20$ & $1.39 \pm 0.1$ \\
\hline ot60 & Dwarf Opal, Companion & $30.8 \pm 2.2$ & $449 \pm 150$ & $69.7 \pm 22.4$ & $1.05 \pm 0.4$ \\
\hline
\end{tabular}

${ }^{\mathrm{a}}$ ot $=$ ocimum accessions numbers.

${ }^{\mathrm{b}}$ All values reported as an average of three replicates \pm SD. 
Table 2. Chemical composition of Ocimum americanum accessions, grown under field conditions (Purdue University)

\begin{tabular}{|c|c|c|c|c|}
\hline \multirow{2}{*}{$\begin{array}{l}\text { Volatile oil } \\
\text { constituents }^{\mathrm{a}}\end{array}$} & \multirow[t]{2}{*}{$\mathrm{RT}^{\mathrm{b}}$} & \multicolumn{2}{|c|}{ Var. americanum } & \multirow{2}{*}{$\frac{\text { Var. pilosum }}{\text { ot21 }}$} \\
\hline & & ot5 & ot32 & \\
\hline & & \multicolumn{3}{|c|}{$\left(\right.$ Relative percentage of total volatile oil) ${ }^{\mathrm{c}}$} \\
\hline 1,8-Cineole & 6.08 & $25.6 \pm 6.3$ & & $15.5 \pm 12.8$ \\
\hline Linalool & 8.1 & $4.7 \pm 1$ & & $1.7 \pm 1.1$ \\
\hline Camphor & 9.24 & $1.4 \pm 0.5$ & & $1.05 \pm 0.2$ \\
\hline Methylchavicol & 11.72 & $0.4 \pm 0.3$ & & $10.4 \pm 5.3$ \\
\hline Anisole & 14.08 & $42.9 \pm 5.7$ & & \\
\hline Methyl(Z)-cinnamate & 16.68 & & $9.65 \pm 0.4$ & \\
\hline Eugenol & 18.72 & $0.4 \pm 0.3$ & & $1.6 \pm 0.3$ \\
\hline $\operatorname{Methyl}(E)$-cinnamate & 20.86 & & $86.5 \pm 0.7$ & $0.6 \pm 0.3$ \\
\hline$\beta$-Caryophyllene & 20.58 & $1.5 \pm 0.6$ & & $0.7 \pm 0.4$ \\
\hline$\alpha$-Bergamotene & 21.24 & & & $5.2 \pm 2.4$ \\
\hline$\alpha$-Humulene & 22.04 & $11.5 \pm 4$ & & $1.8 \pm 0.5$ \\
\hline$\beta$-Bisabolene & 24.58 & & & $29.2 \pm 9.2$ \\
\hline$\alpha$-Farnesene & 25.74 & & & $1.6 \pm 0.5$ \\
\hline Spathulenol & 27.6 & & & $5.8 \pm 2.2$ \\
\hline
\end{tabular}

${ }^{a}$ Listed in order of retention time $(\mathrm{min})$.

${ }^{\mathrm{b}} \mathrm{RT}=$ retention time.

${ }^{c}$ Values reported as an average of three replicates \pm SD.

ot5 $=$ O. americanum $(\mathrm{Kew})$, ot $32=$ O. americanum $($ Brazil $)$.

concentration of $78 \%$ in $O . \times$ citriodorum using solvent extraction method. Extraction methods for citral can lead to significant differences in citral recovery.

Ocimum americanum var. americanum (ot32 and ot5) exhibited the highest volatile oil yield per plant (1.68 and $2.47 \%$, respectively), while the $O$. americanum var. pilosum (ot 21$)$ produced the lowest oil $(0.27 \%$; Table 1$)$. Ocimum basilicum accessions exhibited a range in oil production from 0.58 to $1.64 \%$.

Comparing the volatile oil constituents between the different varieties of $O$. americanum (Table 2), $O$. americanum var. americanum and $O$. americanum var. pilosum showed a completely distinct oil profile. We observed two different chemotypes in the first variety, anisole-rich (ot5) and a methylcinnamate-rich (ot32) chemotypes. The botanical variety pilosum contained $\beta$ bisabolene and methylchavicol as its major constituents. This is the first time $O$. americanum var. pilosum volatile oil has been chemically described, but more accessions will have to be studied to see whether this is a characteristic essential oil profile for var. pilosum.

Ocimum americanum appears to be the most chemically variable species in the genus, with four chemotypes described in the literature. Distinct chemotypes of Ocimum americanum have been reported, such as a citral type in India, ${ }^{24}$ a camphor type from Africa, ${ }^{25}$ and a methyl $(E)$-cinnamate chemotype in Brazil. ${ }^{4}$ Examination of a methyl $(E)$-cinnamate chemotype of $O$. americanum showed no linkage between morphology and chemical compounds, since other methyl $(E)$-cinnamate types were also found in $O$. basilicum.

A total of 21 volatile oil constituents were detected in $O$. basilicum (Table 3). The major constituents detected in our samples included 1,8-cineole, linalool, methylchavicol and methyl $(E)$-cinnamate, all previously described. ${ }^{26}$

The analysis of $O$. basilicum accessions showed mostly a variation of linalool-methylchavicol types, with only two accessions (ot49 and ot71; Table 4) rich in methyl $(E)$-cinnamate. Two additional accessions of $O$. basilicum (ot66 and ot70; Table 3) were considered distinct from other $O$. basilicum accessions. Although morphologically described as $O$. basilicum, 'Sweet Dani Lemon Basil' (ot70) and 'Lemon Mrs Burns' (ot66) exhibited an oil profile similar the generic lemon basil $(O . \times$ citriodorum $)$, and thus appeared to be chemically closer to $O . \times$ citriodorum. These findings are supported by genetic fingerprinting of this species using RAPDs, which placed all citral-rich basils as $O . \times$ citriodorum rather than as $O$. basilicum. ${ }^{15}$

A detailed analysis of $O$. basilicum varieties, showed that 'Cinnamon' basil (ot49) and the Purdue methyl $(E)$ cinnamate chemotype (ot71) can be differentiated by the amount of methyl $(E)$-cinnamate in their oil, with 34 and $82 \%$, respectively. 'Cinnamon' basil appears to have been introduced to USA from Mexico, as 'Spicy Mexican' basil, and was the first flavored variety to be sold by a large US seed company. ${ }^{6}$ The methyl $(E)$-cinnamate chemotype (ot71) was developed over a 10 year breeding project by Purdue University at the Center for New Crops and Plant Products.

The $O$. basilicum accessions 'Dark Opal' (ot38) and 'Purple Ruffles' (ot40) showed similar volatile oil profiles, although the first exhibited a higher linalool content, and the second a higher methylchavicol content. 'Purple Ruffles' is a cross between 'Dark Opal' and 
Table 3. Chemical composition of Ocimum basilicum accessions, grown under field conditions (Purdue University)

\begin{tabular}{|c|c|c|c|c|c|c|c|c|}
\hline Volatile oil constituents ${ }^{a}$ & $\mathrm{RT}^{\mathrm{b}}$ & ot $2^{c}$ & ot38 & ot40 & ot41 & ot43 & ot44 & ot45 \\
\hline & \multicolumn{8}{|c|}{ (Relative percentage of total volatile oil) $^{\mathrm{d}}$} \\
\hline$\alpha$-Pinene & 4.79 & & $1.1 \pm 0.7$ & $1.7 \pm 1.6$ & $1.5 \pm 0.7$ & $0.6 \pm 0.4$ & $0.4 \pm 0.1$ & $0.1 \pm 0$ \\
\hline$\beta$-Pinene & 4.96 & & $0.8 \pm 0.47$ & $1.9 \pm 1.5$ & $1.5 \pm 0.5$ & $0.4 \pm 0.1$ & $0.4 \pm 0.17$ & $0.3 \pm 0.14$ \\
\hline 1,8-Cineole & 6.08 & $1.4 \pm 0.76$ & $11 \pm 4$ & $14.7 \pm 8.5$ & $14.8 \pm 2.3$ & $7.8 \pm 4.4$ & $8.4 \pm 1.8$ & $2 \pm 1.1$ \\
\hline$\gamma$-Terpineol & 7.31 & $0.5 \pm 0.6$ & $0.9 \pm 0.43$ & $0.9 \pm 0.81$ & $0.5 \pm 0.3$ & $1 \pm 0.3$ & $0.9 \pm 0.52$ & $0.8 \pm 0.42$ \\
\hline Linalool & 8.1 & $0.6 \pm 0.8$ & $69.3 \pm 5.6$ & $38.1 \pm 8.2$ & $20.3 \pm 3.4$ & $34.1 \pm 5.6$ & $55.7 \pm 19.3$ & $70.6 \pm 6.2$ \\
\hline Camphor & 9.24 & & & & $2.9 \pm 0.3$ & $0.2 \pm 0.3$ & $0.3 \pm 0.32$ & \\
\hline Terpin-4-ol & 10.77 & & & & $0.5 \pm 0.2$ & $0.4 \pm 0.3$ & $0.6 \pm 0.57$ & $0.2 \pm 0.21$ \\
\hline Methylchavicol & 11.72 & $87.8 \pm 5.8$ & $1.3 \pm 0.3$ & $12.7 \pm 18.6$ & $41.5+2.2$ & $41.7 \pm 5.5$ & $16.4 \pm 20.1$ & $2.6 \pm 0.21$ \\
\hline $\operatorname{Methyl}(Z)$-cinnamate & 16.68 & & & & & & & \\
\hline Eugenol & 18.72 & & $0.6 \pm 0.29$ & $0.4 \pm 0.4$ & $0.3 \pm 0.2$ & $0.1 \pm 0.1$ & $1.8 \pm 3.1$ & $3.5 \pm 1.3$ \\
\hline$\beta$-Elemene & 19.44 & $0.5 \pm 0.25$ & $1 \pm 0.26$ & $1.9 \pm 1.6$ & $0.7 \pm 0.2$ & $0.9 \pm 0.3$ & $1.1 \pm 0.6$ & $0.9 \pm 0.7$ \\
\hline $\operatorname{Methyl}(E)$-cinnamate & 20.45 & & & & & & & \\
\hline Methyleugenol & 20.58 & $0.9 \pm 0.92$ & $0.6 \pm 0.06$ & $1.1 \pm 0.5$ & $0.2 \pm 0.1$ & $0.1 \pm 0.1$ & $0.2 \pm 0.08$ & $0.2 \pm 0$ \\
\hline$\beta$-Caryophyllene & 20.86 & $1.3 \pm 1.14$ & & & & & & \\
\hline$\gamma$-Elemene & 21.24 & $1.3 \pm 0.4$ & $1.6 \pm 0.92$ & $10.9 \pm 3.9$ & $3.6 \pm 0.5$ & $2.4 \pm 0.68$ & $1.6 \pm 0.65$ & $4.4 \pm 2.5$ \\
\hline$\alpha$-Humulene & 22.04 & & $0.5 \pm 0.1$ & $1.1 \pm 0.6$ & $0.6 \pm 0.1$ & $0.4 \pm 0.15$ & $0.5 \pm 0.27$ & $0.6 \pm 0.1$ \\
\hline$\beta$-Cubebene & 23.2 & & $0.8 \pm 0.34$ & $1.8 \pm 0.6$ & $1.4 \pm 0.1$ & $1.4 \pm 0.26$ & $1 \pm 0.5$ & $1.2 \pm 0.6$ \\
\hline$\beta$-Bisabolene & 24.58 & & $1.2 \pm 0.5$ & $1.7 \pm 0.2$ & $1.4 \pm 0.2$ & $1.5 \pm 0.3$ & $1.7 \pm 0.57$ & $1.6 \pm 0.5$ \\
\hline$\alpha$-Farnesene & 25.74 & & & & & & & \\
\hline$\alpha$-Gurjenene & 26.66 & & & & & & & \\
\hline Spathulenol & 27.6 & $1 \pm 0.7$ & $1.0 \pm 0.32$ & $1.3 \pm 0.2$ & $0.3 \pm 0.1$ & $0.5 \pm 0.06$ & $0.9 \pm 0.6$ & $1.1 \pm 0.35$ \\
\hline$\alpha$-Pinene & 4.79 & $0.3 \pm 0.26$ & $0.2 \pm 0.06$ & & & & & \\
\hline$\beta$-Pinene & 4.96 & $0.3 \pm 0.32$ & $0.1 \pm 0.08$ & & & & & \\
\hline 1,8-Cineole & 6.08 & $2.9 \pm 1.6$ & $1.7 \pm 0.66$ & $0.9 \pm 0.65$ & $0.3 \pm 0.19$ & $3.7 \pm 2.7$ & $0.7 \pm 0.38$ & \\
\hline$\gamma$-Terpineol & 7.31 & $2.1 \pm 1.7$ & $0.7 \pm 0.46$ & $\mathrm{t}$ & $0.5 \pm 0.54$ & $0.6 \pm 0.25$ & $2.6 \pm 1.54$ & \\
\hline Linalool & 8.1 & $32.3 \pm 13.1$ & $1.4 \pm 0.6$ & $0.8 \pm 0.15$ & $0.6 \pm 0.36$ & $1.7 \pm 0.49$ & $74.5 \pm 1.7$ & \\
\hline Camphor & 9.24 & $0.9 \pm 0.28$ & $4.3 \pm 0.88$ & $3 \pm 2.04$ & & & & \\
\hline Terpin-4-ol & 10.77 & $0.8 \pm 0.32$ & $1 \pm 0.29$ & $0.8 \pm 0.53$ & & & $0.3 \pm 0.1$ & \\
\hline Methylchavicol & 11.72 & $6.4 \pm 10.8$ & $74.4 \pm 3.4$ & $57.2 \pm 38.2$ & $86.4 \pm 3.8$ & $0.4 \pm 0.1$ & $0.4 \pm 0.15$ & \\
\hline $\operatorname{Methyl}(Z)$-cinnamate & 16.68 & $5.5 \pm 3.7$ & & & & $5.9 \pm 0.75$ & & \\
\hline Eugenol & 18.72 & $1 \pm 2$ & & & $0.1 \pm 0.2$ & & $0.8 \pm 0.62$ & \\
\hline$\beta$-Elemene & 19.44 & & $0.3 \pm 0.3$ & & $0.8 \pm 0.48$ & & $1.2 \pm 0.22$ & \\
\hline $\operatorname{Methyl}(E)$-cinnamate & 20.45 & $34 \pm 22.8$ & $1.0 \pm 0.13$ & $0.8 \pm 0.55$ & & $82.4 \pm 4.2$ & & \\
\hline Methyleugenol & 20.58 & & & & $2.5 \pm 0.75$ & & & \\
\hline$\beta$-Caryophyllene & 20.86 & & & & & & & \\
\hline$\gamma$-Elemene & 21.24 & $0.4 \pm 0.8$ & $4.5 \pm 0.64$ & $3.1 \pm 2.08$ & $0.8 \pm 0.29$ & & $1.9 \pm 0.3$ & \\
\hline$\alpha$-Humulene & 22.04 & $0.3 \pm 0.6$ & $0.2 \pm 0.1$ & $0.3 \pm 0.41$ & $0.8 \pm 0.24$ & & $0.3 \pm 0.21$ & \\
\hline$\beta$-Cubebene & 23.2 & $1.7 \pm 2.26$ & $0.5 \pm 0.22$ & $0.7 \pm 0.95$ & $1.7 \pm 0.52$ & & $2.3 \pm 0.74$ & \\
\hline$\beta$-Bisabolene & 24.58 & $1.9 \pm 1.12$ & $1.4 \pm 0.21$ & $0.9 \pm 0.65$ & $0.2 \pm 0.2$ & $0.2 \pm 0.25$ & $2.5 \pm 0.56$ & \\
\hline$\alpha$-Farnesene & 25.74 & & $1.3 \pm 0.1$ & $1 \pm 0.73$ & $2 \pm 0.69$ & & & \\
\hline$\alpha$-Gurjenene & 26.66 & $1.1 \pm 0.78$ & $0.2 \pm 0.21$ & & $0.1 \pm 0.2$ & & & \\
\hline Spathulenol & 27.6 & $1.3 \pm 0.27$ & $1.6 \pm 0.58$ & $1.2 \pm 1$ & $0.7 \pm 0.22$ & $0.9 \pm 0.42$ & $0.9 \pm 0.1$ & \\
\hline
\end{tabular}

${ }^{\mathrm{a}}$ Listed in order of retention time $(\mathrm{min}) ;{ }^{\mathrm{b}} \mathrm{RT}=$ retention time.

' ot 2 = O. basilicum (Kew); ot38 = 'Dark Opal'; ot40 = 'Purple Ruffles'; ot41 = 'Green Ruffles'; ot43 = 'Mammoth'; ot44 = 'Sweet'; ot45 = 'Sweet Fine'; ot49 = 'Cinnamon'; ot56 = 'Thai'; ot57 = 'Thai Siam Queen'; ot69 = 'Comoro'; ot71 = 'Purdue methyl $(E)$-cinnamate'; ot72 = 'Purdue linalool'.

${ }^{\mathrm{d}}$ Values reported as an average of three replicates \pm SD.

'Green Ruffles' (ot41), which also showed high levels of methylchavicol (41.5\%).

The constituent profile in culinary basils is a very important character. Among the green basils, 'Green Ruffles' (ot41), 'Mammoth' (ot43), 'Sweet' (ot44) and 'Sweet Fine' (ot45) contained 1,8-cineole, linalool and methylchavicol as the major aromatic compounds. The ratio of 1,8-cineole-linalool-methylchavicol varied by variety $(15: 20: 42,8: 34: 42,8: 56: 16$ and $2: 70: 3$, respectively), contributing to their particular aromas.

Linalool chemotype (ot72) had distinct leaf shape compared with 'Sweet Fine' (ot45), although both are linalool-rich. The varieties 'Thai' (ot56), 'Thai Siam Queen' (ot57) and 'Comoro' (ot69) had the highest levels of methylchavicol. One accession introduced from the Royal Botanical Garden at Kew, UK (ot2), and also a methylchavicol type, was morphologically distinct from all other methylchavicol basils.

Of the $O . \times$ citriodorum accessions (Table 5) analyzed, including 'Lemon Mrs Burns' (ot61) and 'Sweet Dani Lemon Basil' (ot70), all showed citral as their major constituent, except for the New Guinea (ot62), a methylchavicol type with no citral. 'Lemon Mrs Burns' (ot61) differs from the others citral types by its high linalool content (46\%).

Of the three accessions of $O$. minimum, 'Bush' (ot34), 'Spice Globe' (ot35) and 'Dwarf Opal' (ot60; Table 5), the first two are green dwarf varieties, while the third is a dwarf purple. All contained linalool as their major constituent (46, 46 and $67 \%$, respectively), followed by 
Table 4. Chemical composition of Ocimum $\times$ citriodorum accessions, and lemon-scented O. basilicum, grown under field conditions (Purdue University)

\begin{tabular}{|c|c|c|c|c|c|c|c|}
\hline Volatile oil constituents ${ }^{a}$ & $R T^{b}$ & ot53 & ot58 & ot61 & ot62 & ot66 & ot70 \\
\hline \multicolumn{8}{|c|}{ (Relative percentage of total volatile oil) } \\
\hline$\beta$-Pinene & 4.96 & $0.1 \pm 0.1$ & $0.3 \pm 0.16$ & $0.5 \pm 0$ & & $0.23 \pm 0.1$ & $0.55 \pm 0.06$ \\
\hline 1,8-Cineole & 6.08 & $0.5 \pm 0.4$ & $0.5 \pm 0.34$ & $2.4 \pm 0.68$ & $2.4 \pm 0.5$ & $0.4 \pm 0.19$ & $0.4 \pm 0.15$ \\
\hline$\gamma$-Terpineol & 7.31 & $1.6 \pm 0.13$ & $2.1 \pm 0.39$ & $3.4 \pm 0.3$ & & $2 \pm 0.24$ & $1.6 \pm 0.61$ \\
\hline Linalool & 8.1 & $8.9 \pm 1$ & $10.7 \pm 1$ & $46.1 \pm 3.9$ & $0.6 \pm 0.59$ & $10.2 \pm 1$ & $1.1 \pm 0.4$ \\
\hline Camphor & 9.24 & $0.3 \pm 0.17$ & $0.3 \pm 0.19$ & $0.6 \pm 0.16$ & & $0.2 \pm 0.24$ & $0.5 \pm 0.1$ \\
\hline Terpin-4-ol & 10.77 & $0.6 \pm 0.32$ & $0.6 \pm 0.13$ & $0.5 \pm 0.06$ & $1 \pm 0.1$ & $0.6 \pm 0.06$ & $0.7 \pm 0.05$ \\
\hline Methylchavicol & 11.72 & $3.7 \pm 0.68$ & $3.7 \pm 0.22$ & $6.4 \pm 0.27$ & $84 \pm 3.6$ & $4.3 \pm 0.75$ & $4.4 \pm 2$ \\
\hline Citral (neral + geranial) & $13.2+14.4^{\mathrm{f}}$ & $31.3 \pm 4.2$ & $29 \pm 1$ & $16.6 \pm 2.6$ & & $32.3 \pm 8.5$ & $33.6 \pm 2.4$ \\
\hline Thymol & 16.42 & $3 \pm 0.6$ & $5.5 \pm 1.5$ & $2.4 \pm 0.9$ & & $2.9 \pm 1$ & $4.4 \pm 1.7$ \\
\hline$\beta$-Elemene & 19.44 & $3 \pm 0.9$ & $1.9 \pm 0.53$ & $1.2 \pm 0.22$ & & $2.7 \pm 0.75$ & $2.3 \pm 0.3$ \\
\hline $\operatorname{Methyl}(E)$-cinnamate & 20.45 & $1 \pm 1.1$ & $1.8 \pm 1$ & $0.6 \pm 0.69$ & $1 \pm 0.32$ & $0.8 \pm 1.3$ & $0.6 \pm 0.08$ \\
\hline$\beta$-Caryophyllene & 20.86 & $3 \pm 0.41$ & $2.4 \pm 0.79$ & $1.1 \pm 0.22$ & $0.6 \pm 0.19$ & $2.8 \pm 0.52$ & $3.1 \pm 0.53$ \\
\hline$\gamma$-Elemene & 21.26 & $28 \pm 0.57$ & $2.6 \pm 0.51$ & $1.9 \pm 0.34$ & $0.6 \pm 0.3$ & $2.8 \pm 0.54$ & $2.4 \pm 0.29$ \\
\hline$\alpha$-Humulene & 22.04 & $1.2 \pm 0.18$ & $0.9 \pm 0.25$ & $0.5 \pm 0.1$ & $0.3 \pm 0.15$ & $1 \pm 0.25$ & $1.3 \pm 0.39$ \\
\hline$\beta$-Cubebene & 23.2 & $1.3 \pm 0.24$ & $0.9 \pm 0.4$ & $0.7 \pm 0.06$ & $0.8 \pm 0.18$ & $0.9 \pm 0.26$ & $7.1 \pm 0.57$ \\
\hline$\beta$-Bisabolene & 24.58 & & & & $1.2 \pm 0.31$ & & \\
\hline$\alpha$-Farnesene & 25.74 & $2 \pm 0.51$ & $1.7 \pm 0.6$ & $0.9 \pm 0.33$ & $0.1 \pm 0.15$ & $2.1 \pm 0.21$ & $2.5 \pm 0.33$ \\
\hline Spathulenol & 27.6 & $15 \pm 1.9$ & $13.4 \pm 1$ & $2.7 \pm 0.5$ & $0.7 \pm 0.26$ & $14.3 \pm 0.4$ & $11.3 \pm 0.9$ \\
\hline
\end{tabular}

${ }^{\mathrm{a}}$ Listed in order of retention time; ${ }^{\mathrm{b}} \mathrm{RT}=$ retention time (min).

' ot53 = 'Lemon' (Richters); ot58 = 'Lemon' (Companion); ot61 = 'Lemon Mrs. Burns', ot62 = 'New Guinea'; ot66 = 'Lemon' (Nichols); ot70 = 'Sweet Dani'.

${ }^{\mathrm{d}}$ formerly $O$. basilicum, included here for its citral composition. ${ }^{\mathrm{e}}$ Values reported as an average of three replicates $+\mathrm{SD}$.

${ }_{\mathrm{f}}^{\mathrm{f}}$ Retention times for neral and geranial, respectively.

Table 5. Chemical composition of Ocimum minimum and O. kilimandscharicum accessions, grown under field conditions (Purdue University)

\begin{tabular}{|c|c|c|c|c|c|c|}
\hline \multirow[b]{2}{*}{ Volatile oil constituents ${ }^{a}$} & \multicolumn{4}{|c|}{ Minimum } & \multicolumn{2}{|c|}{ Kilimandscharicum } \\
\hline & $\mathrm{RT}^{\mathrm{b}}$ & ot34 & ot35 & ot60 & ot3 & ot50 \\
\hline \multicolumn{7}{|c|}{${\text { (Relative percentage of total volatile oil })^{\mathrm{d}}}$} \\
\hline$\alpha$-Pinene & 4.79 & $0.4 \pm 0.17$ & $1.9 \pm 0.26$ & $0.8 \pm 0.32$ & & \\
\hline$\beta$-Pinene & 4.96 & $0.5 \pm 0.33$ & $0.6 \pm 0.06$ & $0.2 \pm 0.26$ & $0.8 \pm 0.27$ & \\
\hline 1,8-Cineole & 6.08 & $6.1 \pm 2$ & $16.3 \pm 1.9$ & $1.3 \pm 0.5$ & $4.4 \pm 1.9$ & $10.2 \pm 1.7$ \\
\hline$\gamma$-Terpineol & 7.31 & $2.1 \pm 0.27$ & $1.4 \pm 0.35$ & $1 \pm 0.82$ & & \\
\hline Linalool & 8.1 & $46.4 \pm 4.1$ & $46.6 \pm 2.6$ & $66.9 \pm 16.3$ & $1.2 \pm 0.2$ & $53.1 \pm 2$ \\
\hline Terpinolene & 8.12 & & & & $0.9 \pm 0.3$ & $0.8 \pm 0.4$ \\
\hline Camphor & 9.24 & $6.4 \pm 1$ & $0.6 \pm 0.19$ & $1.1 \pm 0.53$ & $78.3 \pm 5.1$ & $19.3 \pm 0.53$ \\
\hline Terpin-4-ol & 10.95 & $5.1 \pm 2.77$ & $3.1 \pm 0.29$ & & $0.9 \pm 0.2$ & $0.4 \pm 0$ \\
\hline Methylchavicol & 11.72 & $2 \pm 0.29$ & $1.7 \pm 0.17$ & $0.7 \pm 0.37$ & $2.1 \pm 0.22$ & $0.9 \pm 0.06$ \\
\hline Eugenol & 18.72 & $1.8 \pm 0.44$ & $2.9 \pm 0.29$ & $0.5 \pm 0.37$ & $1.8 \pm 0.5$ & $1.2 \pm 0.1$ \\
\hline$\beta$-Elemene & 19.44 & $1.5 \pm 1.37$ & $0.4 \pm 0.4$ & $8.1 \pm 13.9$ & & $1.9 \pm 0.31$ \\
\hline Methyleugenol & 20.03 & $3 \pm 1$ & $5.4 \pm 0.7$ & $2.4 \pm 1$ & $0.4 \pm 0.6$ & $0.7 \pm 0.2$ \\
\hline$\alpha$-Humulene & 22.04 & $1.2 \pm 1$ & $0.5 \pm 0.05$ & $1.1 \pm 0.77$ & & \\
\hline$\beta$-Cubebene & 23.28 & $1 \pm 0.52$ & $2.1 \pm 0.46$ & $2.2 \pm 0.8$ & $0.9 \pm 0.28$ & $1.2 \pm 0.15$ \\
\hline allo-Aromandrene & 24.28 & $2.4 \pm 0.64$ & $1.9 \pm 0.21$ & $1.3 \pm 0.1$ & & \\
\hline$\beta$-Bisabolene & 24.58 & $1.6 \pm 0.61$ & $2 \pm 0.81$ & $1.7 \pm 0.52$ & & \\
\hline Spathulenol & 27.6 & $1.7 \pm 0.59$ & $1.1 \pm 0.25$ & $1.4 \pm 0.49$ & $0.7 \pm 0.2$ & $1.9 \pm 0.3$ \\
\hline
\end{tabular}

${ }^{\mathrm{a}}$ Listed in order of retention time; ${ }^{\mathrm{b}} \mathrm{RT}=$ retention time (min).

' ot34 = 'Bush basil'; ot $35=$ 'Spicy basil'; ot60 = 'Dwarf Opal'; ot $3=$ O. kilimandscharicum $(\mathrm{Kew})$; ot50 = 'African Blue'.

${ }^{\mathrm{d}}$ Values reported as an average of three replicates $+\mathrm{SD}$.

camphor $(6.3 \%)$, an important secondary compound in 'Bush' (ot34), 1,8-cineole (16.3\%) in 'Spice Globe' (ot35) and $\beta$-elemene (8.1\%) in 'Dwarf Opal' (ot60). Two accessions of $O$. kilimansdcharium were observed, the first (ot3) a camphor-rich (78\%) accession, and the second (ot50) a hybrid between $O$. basilicum and $O$. kilimandscharicum rich in linalool (53\%) and camphor (19\%).
A comparative analysis of the 27 identified compounds by PCA allowed us to select the best distinctions based upon seven major compounds. The PCA allowed us to examine the correlation of each compound, and the contribution of each compound (eigenvalue) in the discrimination of each accession (Fig. 1).

The first three principal components (PC) corresponded to $74 \%$ of the total variation (Table 6). The spatial 


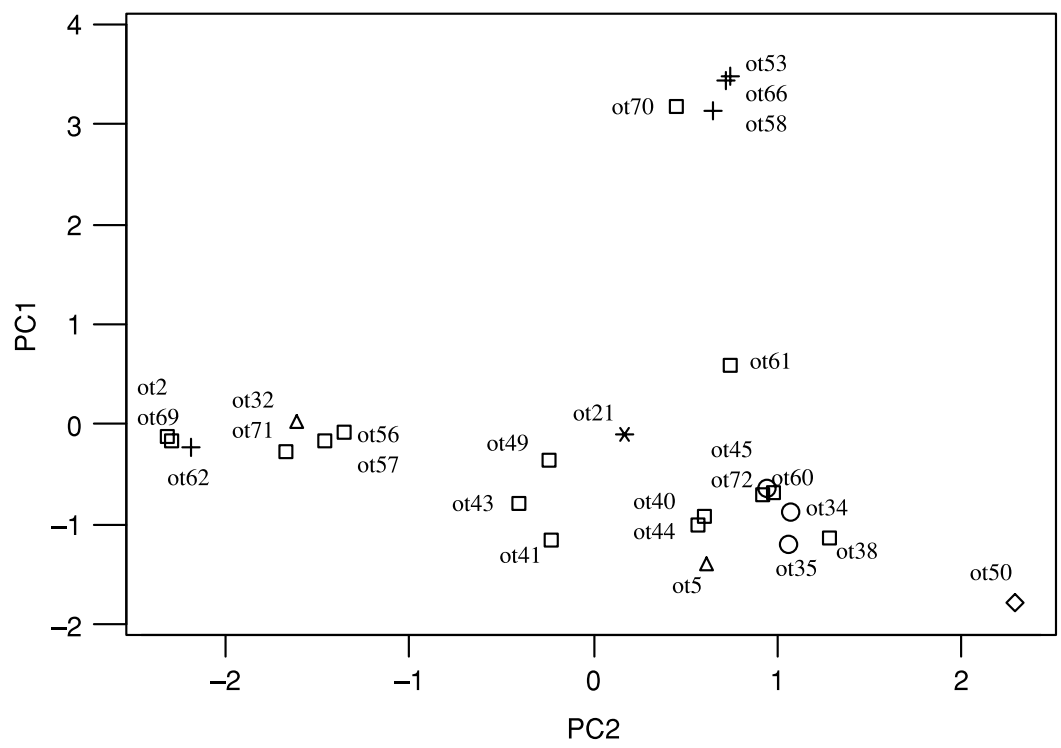

Figure 1. Principal component analysis of seven volatile oil constituents in 26 Ocimum accessions. ( $\square$ ) Ocimum basilicum; (*) O. americanum var. pilosum; $(\triangle)$ O. americanum var. americanum; (+) 0 . citriodorum; $(\bigcirc)$ O. minimum; $(\diamond)$ O. basilicum $\times 0$. kilimandscharicum

Table 6. Eigenvalues and loadings on principal component analysis of variation of volatile oil constituents in Ocimum accessions

\begin{tabular}{lccr}
\hline & \multicolumn{3}{c}{ Principal axes } \\
\cline { 2 - 4 } & I & II & III \\
\hline Eigenvalue & 2.34 & 1.61 & 1.23 \\
Proportion (\%) & 33 & 23 & 18 \\
Cumulative (\%) & 33 & 56 & 74 \\
Loadings & & & 0.272 \\
1,8-Cineole & -0.346 & 0.545 & -0.059 \\
Linalool & -0.282 & 0.270 & 0.244 \\
Camphor & -0.195 & -0.629 & 0.513 \\
Methylchavicol & -0.079 & 0.195 & 0.069 \\
Citral & 0.620 & -0.278 & -0.804 \\
Methyl(E)-cinnamate & -0.004 & 0.212 & 0.104 \\
Spathulenol & 0.610 & & \\
\hline
\end{tabular}

relationship of the 26 accessions using the first three vectors from this analysis is illustrated in Figure 1. The first component explains $33.4 \%$ of the variation and has an eigenvalue of 2.3, which consists of citral and spathulenol as major constituents (Table 6). Citral and spathulenol showed a correlation value of $94 \%$ (data not shown). The PC2 explains $23 \%$ of the variation, with an eigenvalue of 1.6. The $\mathrm{PC} 2$ is bipolar with linalool contrasting with methylchavicol. Both compounds showed a negative correlation of $47 \%$. The PC3 accounts for $17.5 \%$ of the variation, and has an eigenvalue of 1.2 , with methyl $(E)$ cinnamate as the major component of this vector. The PC vectors were able to separate the accessions in five groups, which do not consist of five groups of species studied, based on the major volatile oils constituents: (1) citral-spathulenol-rich accessions; (2) linalool-rich accessions; (3) methylchavicol accessions; (4) linaloolmethylchavicol accessions; and (5) methyl $(E)$-cinnamate accessions (Figure 1).

The use of secondary metabolites in plant taxonomy is well recognized, ${ }^{27,28}$ and often can provide useful characteristics for taxonomic classification. Multivariate analysis techniques have been used successfully in the study of infraspecific ${ }^{18,29}$ and interspecific variability of essential oils. ${ }^{30,31}$

Grayer et $a .^{23}$ reported that the use of one single volatile oil to describe a chemotype is problematic, since there are often two or more major compounds, which might be present in nearly equal amounts and many minor compounds. A system of chemical descriptors based on the combination of major compounds (those with higher than 20\%) rather than the sole dominant compounds was instead proposed by Grayer et al. ${ }^{23}$

The large genetic variation within Ocimum species continues to show a segregation of several minor compounds, including many of the sesquiterpenes, making the use of essential oils that are difficult to establish as a reliable general descriptor for all accessions and varieties. Although chemotypes have been found mainly among the monoterpenes, sesquiterpenes have been shown to play a significant role in the establishment of chemotypes in other species such as Thymus species. ${ }^{18}$

Chemotaxonomic conclusions using solely volatile oils profiles need to be tempered when addressing both a partially domesticated and highly polymorphic group such as Ocimum. It is noteworthy that most studies on chemosystematics using essential oils are made on natural populations, ${ }^{29}$ while many of the basils, particularly the $O$. basilicums, have been subject to some form 
of domestication over a long period. ${ }^{4}$ The use of essential oils as a taxonomic descriptor coupled with genetic fingerprinting ${ }^{14,15}$ would provide in basil a potent combination for taxonomic classifications of this genus.

The groups of Ocimum species based on morphological characteristics do not correspond to the groups based on volatile oils constituents. Each group of volatile oil profiles can occur in different species, for example, the citral/spathulenol type occurs in both $O . \times$ citriodorum and $O$. basilicum; linalool-rich types are frequent in $O$. basilicum varieties, $O$. minimum, $O$. kilimandscharicum and $O . \times$ citriodorum. The presence of methyl-cinnamate-rich types in both $O$. americanum and $O$. basilicum clearly shows that species and essential oil profiles do not necessarily correspond. Basil cultivars are the result of many years of breeding and selection. As morphological traits and chemical characteristic segregation are not necessarily linked, plants can obtain morphological characteristics from one parent or chemical characteristics from the other. Therefore, several variations can be found at infraspecific level.

Acknowledgments-This project was funded in part by the Brazilian Agricultural Research Corporation (Embrapa), Purdue University and the New Use Agriculture and Natural Plant Products Program at Rutgers University.

\section{References}

1. Simon JE, Quinn J, Murray RG. Advances in New Crops Research, Janick J, Simon JE (eds). Timber Press, Portland, Oregon, 1990: 484-489.

2. Albuquerque UP, Andrade LHC. Anales Jard. Bot. Madrid, 1998; 6(1): 43-64.

3. Gupta R. G-15 Gene Banks Med. Arom. Plants Newsl.; 1994; 5/6: $1-3$.

4. Vieira RF, Simon JE. Econ. Bot., 2000; 54(2): 207-216.

5. Simon JE, Chadwick AF, Craker LE. Herbs: an Indexed Bibliography 1971-1980; the Scientific Literature on Selected Herbs, and Aromatic and Medicinal Plant of the Temperate Zone. Archon Books, Hamden, 1984; 7-9.

6. de Baggio T, Belsinger S. Basil, an Herb Lover's Guide. Interweave Press, Colorado, 1996.

7. Darrah H. The Cultivated Basils. Buckeye, Missouri, 1980.

8. Darrah H. Econ. Bot., 1984; 28: 63-67.

9. Marotti M, Piccaglia R, Giovanelli E. J. Agric. Food Chem., 1996; 44: 3926-3929.

10. Harley MM, Paton A, Harley RM, Cade PG. Grana, 1992; 31: 161-176.

11. Paton A. Kew Bull., 1992; 47(3): 403-435

12. Tucker AO. Herbs, Spices and Medicinal Plants., Vol I, Craker LE, Simon JE (eds). Haworth Press, New Jersey, 1986; 33-80.

13. Paton A, Putievsky E. Kew Bull., 1996; 51(3): 1-16.

14. Vieira RF, Grayer R, Paton A, Simon JE. Biochem. Syst. Ecol., 2001; 29: 287-304

15. Vieira RF, Goldsbrough P, Simon JE. J. Am. Soc. Hort. Sci., 2003; 128(1): 94-99.

16. Maffei M. Biochem. Syst. Ecol., 1990; 18(7/8): 493-502.

17. Maffei M, Paracino V, Sacco T. Acta Hort., 1993; 330: 159-169.

18. Canigueral S, Vila R, Vicario G, Tomas X, Adzet T. Biochem. Syst. Ecol., 1994; 22(3): 307-315.

19. Keskitalo M, Linden A, Valkonen JPT. Theor. Appl. Genet., 1998; 96(8): 1141-1150.

20. Adams RP, Demeke T, Abulfatih HA. Theor. Appl. Genet., 1993; 87: 22-26.

21. Charles DJ, Simon JE. J. Am. Soc. Hort. Sci., 1990; 115(3): 458462.

22. Morales MR, Simon JE. Hortscience, 1997; 32(1): 148-149.

23. Grayer RJ, Kite GC, Goldstone FJ, Bryan SE, Paton A, Putievsky E. Phytochemistry, 1996; 43(5): 1033-1039.

24. Sarin, YK, Agarwal SG, Thappa RK, Singh K, Kapahi BK. J. Essent. Oil Res., 1992; 4: 515-519.

25. Demissew, S. J. Essential Oil Res., 1993; 5(5): 465-479.

26. Lawrence BM. Advances in Labiatae Science, Harley RM, Reynolds T (eds). The Royal Botanical Garden, London, 1992; 399-436.

27. Hegnauer R. Chemotaxonomie der Pflanzen, vol IV. Birkhauser, Basel, 1966; 313-314.

28. Gottlieb OR. Micromolecular Evolution, Systematics, and Ecology: an Essay into a Novel Botanical Discipline. Springer, Berlin, 1982.

29. Egerton-Warburton LM, Ghisalberti EL, Considine JA. Biochem. Syst. Ecol., 1998; 26: 873-888.

30. Cool LG, Hu Z, Zavarin E. Biochem. Syst. Ecol., 1998; 26: 899913.

31. Timmermann BN, McLaughlin SP, Hoffmann J. Biochem. Syst. Ecol., 1987; 4: 401-410. 